\title{
Effects on National Health dentistry of attempts to control NHS expenditure
}

\author{
Past and present attempts to control health service expenditure and its effects on National Health dentistry \\ by N. M. Jessop, E. J. Kay, A. C. Mellor, J. G. Whittle, and A. Jenner Br Dent J 2000; 189: 617-619
}

\section{Objective}

To examine the methods used by policy makers to try to reduce or limit expenditure within the National Health Service.

\section{Method}

A chronological examination of cost related health care policy and its wider impact on society. Comparison of past developments in the NHS with new methods of health care organisation in the United Kingdom (UK). The experiences of two dental Total Purchasing Pilots are used to describe where the dental profession now stands in relation to current NHS developments.

\section{Results}

The introduction of Primary Care Groups (PCGs) constitutes part of a continued effort by health care policy makers to control expenditure on health services. In the PCG system, as in Total Purchasing (TP), a group of primary care practitioners control a finite budget. These groups therefore must decide which health care services should be available to the population they represent. Inevitably such systems result in the rationing of health care, but the decisions are instigated by primary health care professionals rather than by government edict.

\section{Conclusion}

Unless GDPs are allowed, willing and able, to participate in and influence the newly emerging Primary Care Groups and Trusts, dental services may once again be pushed to the fringe of NHS care provision. Without adequate representation, the value of dental care may not be recognised and PCGs may decide to make use of traditional dental resources, to fund other services.

\footnotetext{
In Brief

- There has long been central recognition that resources for primary health care must in some way be limited, as demand inevitably increases as service provision improves.

- The dental profession has witnessed many changes in the process and form of remuneration, none of which have been entirely satisfactory to all concerned.

- Primary Care Groups allow groups of practitioners to control a finite budget in order to commission services for their population.

- GDPs are not represented on Primary Care Groups, but can only aspire to influence commissioning decisions via Oral Health Advisory Groups. There is therefore a danger that dental services may be under - or inadequately resourced.
}

\begin{abstract}
Comment
Whilst economics has a good claim to the title 'The Science That Failed', it is hard to disagree with one of its more common sense tenets: that the demand for a good which is free will be infinite. This awkward fact has been a constant problem in the provision of public services and none more publicly or more contentiously so than in the case of the National Health Service.

In this paper - the first of three by Jessop et al. that examine the place of dentistry in the NHS - the authors chronologically examines attempts to limit the cost of the
\end{abstract}

NHS to the Exchequer, starting with the early use of rigid hierarchical structures to dictate spending, right through to the use of health outcome measures.

They discuss the introduction of fund holding in the 1980s, the reaction to it, and the subsequent move to Total Purchasing (TP). TP is particularly important as it was this structure that was to develop into Primary Care Groups (PCG) and the early experience of the dental practitioners who participated in the Total Purchasing Pilots is discussed in some detail.

Initially, dental services were excluded from TP and although this was modified later under the PCGs the role they were to play was still unclear. Primary Care Trusts have given rise to Oral Health Advisory Groups as a specific measure to bring about the involvement of dentists and the authors point out that unless dentists are willing and able to participate in the process it is likely that dental services will once again be pushed to the edges of NHS care provision.

\section{Richard Foulsham}

Senior Assistant Editor, British Dental Journal 